\title{
Spontaneous reoccurrence of "scooping", a wild tool-use behaviour, in naïve chimpanzees
}

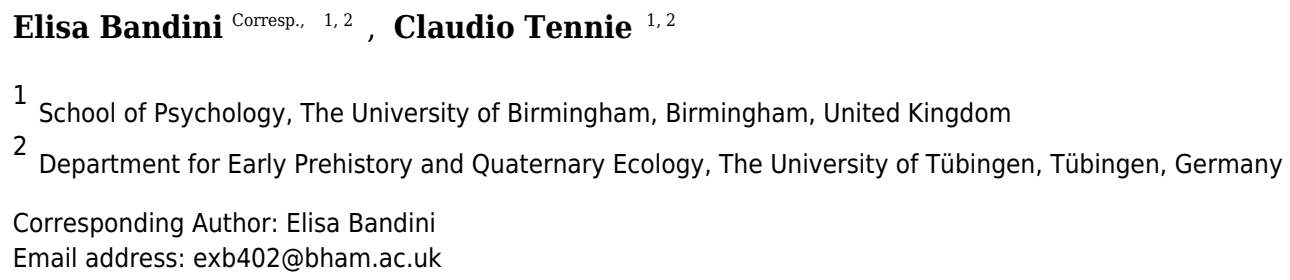

Modern human technological culture depends on social learning. A widespread assumption for chimpanzee tool-use cultures is that they, too, are dependent on social learning. However, we provide evidence to suggest that individual learning, rather than social learning, is the driver behind determining the form of these behaviours within and across individuals. Low-fidelity social learning instead merely facilitates the reinnovation of these behaviours, and thus helps homogenise the behaviour across chimpanzees, creating the population-wide patterns observed in the wild (what here we call "socially mediated serial reinnovations"). This is the main prediction of the Zone of Latent Solutions (ZLS) hypothesis. This study directly tested the ZLS hypothesis on algae scooping, a wild chimpanzee tool-use behaviour. We provided naïve chimpanzees $(n=14$, Mage $=31.33$, $S D=10.09$ ) with ecologically relevant materials of the wild behaviour but, crucially, without revealing any information on the behavioural form required to accomplish this task. This study found that naïve chimpanzees expressed the same behavioural form as their wild counterparts, suggesting that, as the ZLS theory predicts, individual learning is the driver behind the frequency of this behavioural form. As more behaviours are being found to be within chimpanzee's ZLS, this hypothesis now provides a parsimonious explanation for chimpanzee tool cultures. 
1 Full title: Spontaneous Reoccurrence of "Scooping", a Wild Tool-Use Behaviour, in Naïve Chimpanzees

2 Short title: Chimpanzees Reinvent Tool-Use Behaviour

3 Elisa Bandini ${ }^{1,2^{*}}$ and Claudio Tennie ${ }^{1,2}$

$4 \quad{ }^{1}$ The University of Birmingham, School of Psychology, Birmingham, B15 2TT, UK

$5{ }^{2}$ The University of Tübingen, Department for Early Prehistory and Quaternary Ecology, Tübingen,

6 72070, Germany

7

$8 \quad{ }^{*}$ Corresponding author, Elisa Bandini:

9 Address: The University of Birmingham, School of Psychology, Birmingham, B15 2TT, UK

10 Email: exb402@bham.ac.uk 


\section{$12 \quad$ Abstract}

13 Modern human technological culture depends on social learning. A widespread assumption for 14 chimpanzee tool-use cultures is that they, too, are dependent on social learning. However, we provide 15 evidence to suggest that individual learning, rather than social learning, is the driver behind 16 determining the form of these behaviours within and across individuals. Low-fidelity social learning 17 instead merely facilitates the reinnovation of these behaviours, and thus helps homogenise the 18 behaviour across chimpanzees, creating the population-wide patterns observed in the wild (what here 19 we call "socially mediated serial reinnovations"). This is the main prediction of the Zone of Latent 20 Solutions (ZLS) hypothesis. This study directly tested the ZLS hypothesis on algae scooping, a wild 21 chimpanzee tool-use behaviour. We provided naïve chimpanzees $(n=14$, Mage $=31.33, \mathrm{SD}=10.09)$ 22 with ecologically relevant materials of the wild behaviour but, crucially, without revealing any 23 information on the behavioural form required to accomplish this task. This study found that naïve 24 chimpanzees expressed the same behavioural form as their wild counterparts, suggesting that, as the ZLS theory predicts, individual learning is the driver behind the frequency of this behavioural form. As more behaviours are being found to be within chimpanzee's ZLS, this hypothesis now provides a parsimonious explanation for chimpanzee tool cultures. 


\section{Introduction}

32 A growing body of literature suggests that humans are not unique in their possession of culture (culture defined as: "behavioural variation that owes its existence at least in part to social learning processes", Perry, 2006). In fact, various taxonomic groups provide evidence for some such form of culture. For example, whales (Cetacea; Rendell \& Whitehead, 2001), capuchin monkeys (Cebus; Fragaszy et al., 2004), New Caledonian crows (Corvus; Weir \& Kacelnik, 2006), and great apes (McGrew, 1998; Whiten et al., 1999; van Schaik et al., 2003) have all been suggested to have culture. Among all these, great apes, and in particular chimpanzees (Pan troglodytes), are often described as having the most extensive repertoire of cultural behaviours (Sanz \& Morgan, 2007; Whiten \& van Schaik, 2007; Koops et al., 2014). As the challenge to understand how human culture evolved continues (one of the top 125 questions of our time, see Science, special $125^{\text {th }}$ anniversary issue, 2005), particular focus has been placed on chimpanzee culture due to their close phylogenetic ties to modern humans and their potential for providing insight into the evolution of hominin material culture (Tomasello, 1999; Whiten et al., 2009; Tennie et al., 2016 Koops et al., 2015).

The current widespread assumption is that chimpanzee tool-use culture is based on homologous social learning mechanisms to human culture (Kummer \& Goodall, 1985; Boesch, 1996; Whiten et al., 1999; Whiten et al., 2001; De Waal, 2001). Human culture is most likely dependant on high-fidelity social learning mechanisms that transmit information faithfully enough to allow for the cumulative nature of our culture (the so-called ratchet effect; Tomasello, 1999; Tennie et al., 2009). Although the exact mechanisms for this faithful transmission are still debated, imitation (including action copying) and special forms of teaching (imitation-based teaching) are often cited as requirements for the (seemingly) unique aspects of human culture (Tomasello et al., 1993a; Boyd \& Richerson, 2005; Hoppitt et al., 2008; Tennie et al., 2009; Dean et al., 2012; Kline, 2014. Although see also Caldwell \& Millen, 2009; Reindl et 
54 al., 2017, for recent evidence that imitation may not always be necessary for cumulative culture to 55 emerge).

56 Although some claim for evidence of high-fidelity social learning in non-human great apes (Whiten et al., 57 1996; De Waal, 2001; Whiten et al., 2009; Hopper, 2016; Musgrave et al., 2016), the actual data for 58 spontaneous high-fidelity social learning in enriched captive apes, (i.e., apes who live in social groups, 59 and have not been trained by humans, intentionally or unintentionally (not enculturated), see Tennie \& 60 Henrich, in press), remains questionable (Tennie et al., 2009).

61 Indeed, previous studies and observations have failed to show conclusive evidence of action-copying and 62 (imitation-based) teaching in chimpanzees, leading chimpanzees to be categorised as emulators 63 (reproducers of environmental results) rather than spontaneous imitators (where action copying would 64 play a role; Tomasello et al., 1986; Tennie et al., 2006; Tennie et al., 2009; Tennie et al., 2012; Myowa65 Yamakoshi \& Matsuzawa, 2000. Although see also: Whiten et al., 2004; Hopper et al., 2007, Yamamoto 66 et al., 2013). The converse claim (which instead reports that non-human great apes can and do copy 67 actions) stems mainly from the outcomes of so-called 'two-target' tests (e.g. Whiten et al., 1996; Whiten, 68 1998; Whiten et al., 2005; Whiten et al., 2008 for work with chimpanzees, Custance et al., 2001 with 69 orang-utans, and Stoinski et al., 2001 with gorillas). However, using this very kind of task, it was also 70 found that the demonstrations that allow for imitation (demonstrations which include action information, 71 often called "full demonstrations") are not necessary for the observer apes to show the demonstrated 72 target actions - demonstrations of pure environmental results have been shown to lead to copying in this 73 task, too (Hopper et al., 2007). Furthermore, a variety of animals have now been shown to be successful 74 copiers in two-target tests (e.g. pigeons, Lefebvre, 1986; capuchins, Custance et al., 1999; Dindo et al., 75 2008; vervet monkeys, de Waal et al., 2010), and recently even reptiles have been found to copy targets in 76 this kind of task (Kis et al., 2016). Thus, the two-target method does not seem to be measuring any special 77 copying mechanisms (at least not for the presence of any otherwise rare ability that humans may share 78 only with non-human great apes). 
79 Due to the absence of convincing evidence for high-fidelity social learning in non-human great apes, it

80 has been suggested that chimpanzee cultural behaviours must derive their form and stability from

81 processes other than high fidelity copying (or high fidelity teaching; Tennie et al., 2009; Tennie et al.,

82 2006; Moore, 2012). Thus, to truly test whether (any form of) social learning is indeed necessary for the

83 expression of a behavioural form, how the behaviour first emerges needs to be examined. Yet, identifying

84 the first natural occurrence of a behaviour (most often in the wild) is often very difficult. Previous studies

85 have instead attempted to seed behaviours in captive (and sometimes wild) groups to examine how the

86 behaviour spreads across individuals. For example, a recent report on chimpanzee tool-use cultures

87 identified how a behaviour (moss sponging) spread through a population once it naturally occurred

88 (Hobaiter et al., 2014). Although studying how a behaviour emerges across individuals is important,

89 examining the origins of the behaviour can provide valuable insights into the learning mechanisms that

90 are required for its acquisition in an individual - including identifying when behaviour copying is not

91 necessary. In the case of the recent report by Hobaiter and colleagues (2014), the authors argue that social

92 transmission explains $85 \%$ of moss-sponging events in Budongo Forest (Uganda). Whilst we agree that

93 social learning played a role in explaining these increases in frequency of the behaviour, the same data set

94 also showed that moss sponging was independently innovated by at least two individuals in the

95 population (namely the alpha male and alpha female, Hobaiter et al., 2014, p.3). The independent

96 reinnovations of this behaviour demonstrate that individual learning fully accounts for the behavioural

97 form, yet low-fidelity social learning facilitates its frequency across individuals (creating the observed

98 population-wide patterns). In a recent follow-up study by Lamon et al., (2017), the authors discuss the

99 roles of individual and low-fidelity social learning in moss-sponging in Budongo Forest: 'Of course, each

100 moss-sponger has to individually learn the behaviour, but in all likelihood, this was facilitated by the

101 social influence exerted by other group members that acted as model'. We agree. In other words, high

102 fidelity copying does not appear to be strictly necessary to explain the spread of this behaviour across a 
103 population. And, given the growing literature on spontaneous innovations of wild-type behaviours ${ }^{1}$ by 104 naïve individuals across a growing number of animal species (e.g. nest-building in weaver birds (T. $c$. 105 cucullatus), Collias \& Collias, 1964; nut-cracking in capuchins (Sapajus. apella), Visalberghi, 1987;

106 hook-making in New Caledonian crows (Corvus moneduloides), Weir et al., 2002; and functional tool

107 making in Hawaiian crows (C. hawaiiensis), Rutz et al., 2016; nettle-feeding in gorillas (Gorilla beringei

108 beringei), Tennie et al., 2008; leaf-swallowing in chimpanzees (Pan troglodytes) and bonobos (Pan

109 paniscus), Menzel et al., 2013; moss- sponging by (also wild) chimpanzees (Pan troglodytes

110 schweinfurthii), Hobaiter et al., 2014; non-human great ape tool-use by human children (Homo sapiens),

111 Reindl et al., 2016), we argue that the form of tool-use behaviours in great apes appears across individuals

112 by being fuelled by individual learning. In these cases, social learning is not required to explain the form

113 of the underlying behaviour (which instead derives individually), but instead (low-fidelity) social learning

114 facilitates the reappearance of the behaviour across individuals (what we would like to call: "socially

115 mediated serial reinnovations" (SMSR)).

116 Thus, whilst human social learning transmits the form of a behaviour between individuals and

117 consequently spreads the actual behaviour across individuals, other great apes (and possibly all other

118 animals, too) may be forced to continuously 'reinvent the wheel' (metaphorically speaking) due to the

119 forms of their behaviours being largely the products of independent individual learning ${ }^{2}$. If so, such

120 innovations would have to be within the species' potential individual behavioural inventive repertoire,

121 referred to as their 'zone of latent solutions' (ZLS, Tennie et al., 2009). Social learning mechanisms (of a

122 low fidelity type) foster the release of the latent behaviour in others in the population - i.e. may be

123 responsible for the illusion of a seeming spread of a given latent solution - but these mechanisms are not

\footnotetext{
${ }^{1}$ Here we use 'wild-type' behaviours to describe behaviours that are shown by wild non-human populations - including behaviours described as cultural in the literature (e.g. those described by Whiten et al., 1999; 2001).

${ }^{2}$ Note that humans also have a ZLS, i.e. behavioural forms that do not require social learning - but humans can go beyond their ZLS by cumulative culture, which is enabled via their high-fidelity social learning (Reindl et al., 2016). Culture also interacts with human cognition itself, and so this process ultimately leads to ontogenetic cultural intelligence (Tennie \& Over, 2012, and see also Hermann et al., 2007; Reindl et al., 2016).
} 
124 necessary to explain the behavioural form that comes about across different individuals. The ZLS 125 approach thus provides a more parsimonious explanation for chimpanzee behaviours, in which individual 126 learning is hypothesised to be the main motor that drives the frequency of their tool-use behavioural 127 forms as well as explaining the similarity in behavioural form across individuals, rather than assuming 128 that social learning is necessary for the latter (and, especially, without the need to assume high fidelity 129 social learning mechanisms).

130 Still, the behavioural patterns in the wild demonstrate that occasionally even neighbouring communities 131 differ in their behaviour (i.e. where genetic and environmental influences are kept to an absolute 132 minimum - leading to the conclusion that these differences arose and are maintained by social learning; 133 Langergraber et al., 2010). Thus, any theory that attempts to explain chimpanzee behaviour pattern must 134 be able explain how such differences come about. The ZLS hypothesis provides the following 135 explanation. Patterns such as these can be explained by social learning of a low-fidelity type increasing 136 the frequencies of certain latent solutions once they are expressed in the first individual (or, rather 137 simultaneously, in several individuals at once, as was found in Hobaiter et al. 2014). In other words, such

138 social learning processes must increase the likelihood of individual expressions of the latent solutions in 139 question. Thus, once a given latent solution is expressed by the first individual(s), low-fidelity forms of 140 social learning (which are widespread in the animal kingdom) then essentially act to homogenise the

141 likelihood of individual expression of the behaviour within the affected community. In other words, non-

142 human great apes would not so much be specialised in exceptional social learning mechanisms, but they

143 would instead be specialised in increased levels (or: reach) of individual learning. The ZLS hypothesis

144 therefore offers an, at base, individual learning account for the form of behaviours, with low-fidelity 145 social learning acting as a facilitator for the innovation of behavioural forms across connected individuals.

146 The result is the creation of between-population patterns of chimpanzee tool-use behaviours, i.e. what we 147 set out to explain (e.g. population A might show tool-use $\mathrm{X}$, while population B might not). Thus, 148 similarly to accounts that favour a major role for (high-fidelity) social learning, social learning is still 
149 required to explain the patterns of at least some behaviours seen across populations - but it would be of a 150 low-fidelity type (i.e. not transmitting the behaviours themselves).

151 The only way to ascertain whether chimpanzee tool-use is indeed best accounted for by a latent solutions 152 approach is to directly test whether these behaviours can be expressed by naïve individuals (a direct 153 prediction following from the ZLS hypothesis; Tennie et al. 2009). The alternative approach, where high 154 fidelity social learning transmits the behavioural form, would instead predict that these forms cannot be 155 spontaneously shown by individuals that are unconnected to the culture that keeps them in place (and 156 form). In such tests, subjects are considered naïve if they are in this sense unconnected, i.e. they have 157 never been trained in and/or have never seen the behaviour before. To ensure ecological validity, subjects 158 should be so-called enriched captive apes (see Tennie \& Henrich, in press). Subjects are then provided 159 with the necessary raw material and motivation (e.g. food baits) to develop the target behavioural form 160 (this is a latent solutions (LS) test; Tennie et al., 2009). If the naïve subjects develop the target form, this 161 demonstrates that social learning (of either low-fidelity or high-fidelity type) is not necessary for 162 explaining the tested behavioural form (and it becomes unparsimoneous to assume that social learning is 163 responsible for the form in the wild).

164 Data collected from LS studies can then be generalised to a species-level through one of the two ZLS 165 standards (which we will introduce here): the 'single-case ZLS standard' and the 'double-case ZLS 166 standard'. The two standards reflect the varying relative complexity of animal tool-use behaviours. For 167 relatively complex behaviours, such as chimpanzee nut cracking (which requires a specific technique 168 preformed in a predetermined order and several objects in conjunction, Boesch et al., 1994), it is very 169 unlikely that the behaviour is ever shown by pure chance (e.g. during display). Thus, for relatively more 170 complex behaviours we only require a single demonstration of the behaviour in LS tests to conclude that 171 the behavioural form is within the specie's ZLS (i.e. the single-case ZLS standard). Relatively less 172 complex behaviours, such as chimpanzee stick-use, have a slightly higher chance (though still low) of 173 being demonstrated through chance alone. Therefore, for relatively less complex behaviours, we propose 
174 that two individuals must demonstrate the behavioural form independently from one another for it to be 175 concluded to be within the specie's ZLS (i.e. the double-case ZLS standard).

176 To test the Latent Solution Hypothesis we provided naïve captive chimpanzees with all the materials 177 necessary to execute the behavioural form underlying algae scooping behaviour of wild chimpanzees 178 (which we operationalize here as 'scooping', see section below). As is necessary for a latent solution test, 179 we tested the chimpanzees without presenting them first with demonstrations of the target behaviour. 180 Thus, we were able to isolate the roles of learning mechanisms, allowing us to examine whether social 181 learning is necessary for this behavioural form to emerge in chimpanzees. If a tool-use behaviour does 182 rely on social transmission (i.e. where the actual form of the behaviour is, and must be, socially 183 transmitted - as is the case in modern human culture), then it should never occur in circumstances in 184 which social learning is not possible: it should therefore never re-occur in a Latent Solution Test. If 185 instead it is a latent solution, it should re-occur under such conditions (Tennie et al. 2009). Due to the 186 target behaviour being among the relatively less complex behaviours of chimpanzees (a variant of stick187 use), we applied the double-case ZLS standard, and required at least two individuals to independently 188 show the behaviour for it to be classified a latent solution.

\section{Scooping}

190 Algae scooping (not to be confused with 'algae fishing'; Boesch et al., 2016), is a behaviour observed in 191 wild chimpanzees in Bossou, Guinea. The behaviour involves feeding on aquatic algae using herbaceous 192 tools (Humle et al., 2011). These chimpanzees use tools to feed on Spirogyra sp., a common form of 193 algae in Bossou that often covers the surface of ponds, streams and lakes (Humle et al., 2011). Although 194 algae scooping has also been described elsewhere (Sakamaki, 1998; Devos et al., 2002), Humle and 195 colleagues (2011) provide the only description of the actual form of the behaviour. The authors (Humle et

196 al., 2011) divided algae scooping in wild chimpanzees into six steps ${ }^{3}$ : (1) select a stalk or stick, (2) detach

\footnotetext{
${ }^{3}$ Note that whenever a behaviour is divided into steps, one necessarily has to make, to a certain degree, subjective decisions (e.g. on a coarse level, would one include the way from, say, the night nest to the
} 
197 it from the branch or bush, (3) modify its length, (4) remove the leaves, (5) insert it into the water and (6)

198 scoop the algae, using a 'gentle swivelling action of the wrist' (Humle et al., 2011). Our study focused

199 on the behavioural form of scooping and the accompanying actions (steps 1, 5 and especially 6). We

200 concentrated on scooping because the selection, procurement and modification of sticks (steps 1-3) are

201 already known to be widespread behaviours in chimpanzees, strongly suggesting that they can be

202 individually innovated (see Whiten et al., 1999; Gruber et al., 2010 for reviews of tool-use in wild and

203 captive apes). Likewise, we were not interested in how chimpanzees might learn that algae are edible or

204 where they can be found. While such learning can also be, and presumably often is, socially mediated in

205 chimpanzees (e.g., via local and/or stimulus enhancement, see description of social learning terms in

206 Whiten et al., 2004), this kind of information (what and where) does not require the copying of

207 behavioural form from other individuals. Thus, the question of how individuals learn what exactly to do

208 at the location or with the new type of food would remain unanswered. Consequently, when examining

209 whether high or low-fidelity social learning mechanisms are required for animal tool-use behaviour to

210 emerge, logically, the experimental focus must be on the behaviour (the actions) itself. Here we focused

211 on examining the necessary learning mechanisms behind the scooping tool-use actions (identifying the

212 need for a stick, inserting the stick and using it to scoop by applying a 'swivelling action of the wrist') by

213 testing whether they would reappear spontaneously in naïve chimpanzees without the aid of social

214 learning.

215 To recreate the need for a scooping action we provided chimpanzees with floating, elongated bread-crusts

216 out of immediate reach - thus affording a swivelling action with a stick to retrieve the food. Crucially,

217 what "algae scooping" and "bread scooping" have in common is that they require an appropriate stick tool

218 and the target scooping action to retrieve items from a water surface. We tested the ZLS hypothesis

219 (Tennie et al., 2009) on scooping behaviour by providing two groups of naïve, captive chimpanzees

220 housed in a zoo in the United Kingdom with all the ecological requirements and motivation for this

algae as a separate step in the sequence? On a fine level, should one count the movement of single finger digits? Byrne et al.,2001). 
221 behaviour to emerge (appealing floating food that could only be retrieved using sticks in a scooping 222 manner). If at least two of these scooping-naïve chimpanzees spontaneously used sticks to retrieve the 223 floating food with actions similar to the one used by wild chimpanzees, then this would strongly suggest 224 scooping as being a behaviour within chimpanzees' ZLS (following the double-case ZLS standard, see 225 above). To the best of our knowledge, no previous latent solution test has been carried out on the origins 226 of scooping behaviour in chimpanzees or any other non-human great ape.

\section{Materials and Methods}

\section{Terminology}

229 Throughout this manuscript we mention the 'reinnovation' or 'innovation' of wild tool-use behaviours in chimpanzees. We use the term 'reinnovation' when the specific actions (such as 'swivelling' the wrist to scoop algae; Humle et al., 2011) recorded in a wild-type behaviour are observed spontaneously in naïve chimpanzees. Here we follow the definition of innovation provided by Reader \& Laland (2003), in which innovation is: 'a process that results in new or modified learned behaviour and that introduces novel behavioural variants into a population's repertoire'. Crucially, the authors clarify that 'population repertoire is not meant to imply that all individuals in a population will necessarily acquire the novel behaviour, but rather that at least one individual in the population will behave in a manner not previously seen' (Reader \& Laland, 2003). Thus, latent solutions could be described as innovations according to this definition.

Our focus lies on examining whether the form of these innovations in non-human animals derives via non social-processes, and to emphasise the hypothesised individual learning aspect of innovations, we only

241 refer to the very first description ${ }^{4}$ of a behaviour as an 'innovation' but we prefer to call to all subsequent

242 re-occurrences of the same behaviour as reinnovations (e.g. a behaviour is counted as a reinnovation

\footnotetext{
${ }^{4}$ This description can come from wild or captive data - but usually comes from the wild.
} 
243 when a similar form of the behaviour appears in unconnected, naïve individuals (either in captivity - or in

244 the wild (namely when the behaviour is also found in culturally unconnected wild populations)).

\section{Subjects}

246 Fourteen captive chimpanzees, ranging from 7 to 49 years of age (Mage=31.33, SD=10.09), based in a

247 zoo in the United Kingdom took part in this study. All the chimpanzees are housed in social groups and

248 have access to two indoor enclosures and two outdoor enclosures (with observational windows for

249 visitors) and two indoor management areas, which are out of view of visitors. Throughout the enclosure

250 the subjects have access to enrichment apparatuses such as climbing ropes and hanging feeders and are

251 regularly provided with other enrichment devices. Subjects are never deprived of food or water, and

252 continued with their regular feeding routine throughout this study. All subjects participated voluntarily in

253 this study.

254 The chimpanzees were housed in two groups. In Group 1, seven out of the nine chimpanzees were born 255 and raised in captivity (three males and six females, mean age: 27.7 years). In Group 2, four out of five 256 chimpanzees were born and raised in captivity (two males and three females, mean age: 30.8 years (see 257 S1 and S2 tables for more information)). Wild born individuals were originally from the Democratic 258 Republic of Congo or of unknown origins, whilst the majority of the captive born individuals were born 259 at the testing institution. Owing to zoo management requirements, it was not possible to test each 260 individual separately; so they were tested in their normal group settings. The groups are kept separate, and 261 no observation between the two groups was possible during testing. The testing was carried out in their 262 respective communal management areas, and no individual was excluded. This project was reviewed and 263 approved by the University of Birmingham AWERB committee (reference UOB 31213) and by the host 264 zoo following guidelines provided by the SSSMZP, EAZA, BIAZA and WAZA on animal welfare and 265 research in zoological institutions. This study adhered to legal requirements of the UK, where the research 266 was carried out, and adhered to the ASP principles for the Ethical Treatment of Primates. 
267 In order to fully isolate the roles of social and individual learning in a given target behaviour, the subjects

268 must be naïve prior to testing. To test for this, all the keepers were interviewed separately in order to 269 assess whether the chimpanzees had any previous experience with similar tasks, behaviours or materials.

270 We asked for a detailed description of any spontaneous tool-use they may have seen and all past research

271 and enrichment exercises the subjects had participated in that might have been similar to the one

272 presented here (please see supplementary for a summary of the subject's tool-use experience). The

273 keepers independently confirmed that none of the chimpanzees in this study had previously been exposed

274 to any tasks, behaviours or materials similar to the one provided in our current study. The keepers

275 reported that the chimpanzees did have access to sticks before our study, but as our focus was not on

276 general stick use (which is already known to be widespread in great apes and thus reinnovated multiple

277 times; Whiten et al., 1999; 2001) previous contact with sticks did not present a problem to our study.

278 Crucially, the keepers confirmed that the tested subjects were naïve to the problem of having to retrieve

279 out of reach food and to the scooping action. Thus, it is highly unlikely that the subjects in this study,

280 despite having had access to sticks, had previous experience with the problem of retrieving food from a

281 body of water through the use of sticks (there are no water surfaces in the enclosure). Furthermore, the

282 keepers also confirmed, through the questionnaire and in person, that the chimpanzees did not have any 283 experience with the 'swivelling' action required for the scooping behaviour seen in the wild (Humle et al., 284 2011). Although the ideal conditions would involve testing a group of chimpanzees raised in a fully 285 controlled environment, these conditions do not exist to the best of our knowledge, (and would, in any 286 case, lead to ethical problems). Therefore, the best available option involves testing captive chimpanzees 287 whose previous experiences can be confidently accounted for (as we did here).

\section{Procedure}

A square plastic container $(16 \mathrm{~cm} \times 66 \mathrm{~cm} \times 20 \mathrm{~cm})$ was placed outside the enclosure's mesh and filled with room-temperature water. Three bamboo sticks, modelled on the sticks collected in the field (Humle et al.,

291 2011) in Bossou (min. 35cm and max. 98cm long, mean: $66.5 \mathrm{~cm}$ - diameter min. $5 \mathrm{~mm}$, max 30mm, mean: 
$29217.5 \mathrm{~mm}$ ), were placed around the enclosure prior to the chimpanzees entering the management area 293 (again, given our focus on scooping actions, the provision of detached sticks presented no problem to our 294 study design). Prior to testing, the food (bread) was left to harden for a week so that it would float on the 295 top of the water. The bread was cut into 'half-moon' shapes, to allow for it to be retrieved using a 296 scooping action, similarly to algae in the wild. Three pieces of prepared bread pieces (half-moons) per 297 testing session were placed simultaneously in the water container right before testing began. See Fig 1 for 298 the experimental set up.

$299 \quad$ Fig 1.

300 Testing began at around $12.30 \mathrm{pm}$ each day. Once the chimpanzees were allowed into the management 301 area, a ten-minute testing period commenced. Sessions were video recorded on a Sony HDR-CX330E 302 handycam. The test was live coded by E1 (EB) and filmed by E2 (FR). All chimpanzees then had 303 potential access to the apparatus. Each group was tested three times: twice on consecutive days, and then 304 a third time after 28 days. It was live coded whether the subjects used a tool to retrieve the food; if they 305 used a scooping technique (following the description by the Humle et al., 2011, including the target 306 swivelling wrist motion described in the original report) or a different technique; whether there were any 307 instances of stick modification; how the stick was inserted into the bucket; and whether the attempt was 308 successful or not (an attempt was coded as successful if the individual managed to retrieve a piece of 309 bread, including the smaller pieces that formed when the crusts started to disintegrate, and transport it to 310 the mesh).

\section{Results}

\section{Reliability Coding}

313 To assess inter-observer reliability, a naïve individual - who was not familiar to the task or the hypothesis 314 - coded from the videos all the same categories that had been lived coded. These categories were coded 
315 for each attempt in all six videos. The overall Cohen's Kappa was calculated (for a total of 164 instances):

316 there was very good agreement between the two coders, $\mathrm{K}=.870$.

317 Within the first ten minutes of testing (HO: 6 minutes 23 seconds and LO: 7 minutes 9 seconds), two

318 females, HO (33 years, parent-reared and captive born at the testing institution) in Group 1 and LO (37

319 years, hand-reared and captive born at the testing institution) in Group 2, independently retrieved the

320 floating food using stick tools and a scooping action (See S1 file for video clip of individual HO scooping

321 the bread). No other subjects showed these behaviours, but note that, a) throughout the experiment,

322 attempts to use the tools by other members of the group were actively discouraged by HO and LO, who

323 dominated the testing apparatus. Thus, it is possible that other individuals might have used the scooping

324 technique if they had been granted access to the apparatus. And b) because the individuals could not be

325 tested independently, data from individuals other than the first are generally un-interpretable with regard

326 to our research question, as once one subject expresses the behaviour, other individuals can no longer be

327 considered target-naïve. Thus, in a group setting, only the first occurrence per group counts in a Latent

328 Solutions Test, as social learning can no longer be logically excluded afterwards. Given the absence of

329 scooping demonstrations for $\mathrm{HO}$ and LO, as well as their established scooping-naivety at test (see above),

330 these two individuals could not have socially learnt the behaviour, suggesting that both independently

331 reinnovated it.

332 When scooping, $\mathrm{HO}$ and LO would insert the tool into the water above or close to the crusts and then

333 gently rotate the wrist until the bread crust was wrapped around the stick. Once the bread crust was

334 balanced on the tool, it was retracted towards the mesh. See Fig 2 for an example of the scooping 335 technique shown by HO.

336 The reinnovated scooping actions in our study were very similar to the wild scooping behaviour: the wild

337 chimpanzees, as well as the two captive chimpanzees in the current study, scooped using "a gentle 338 swivelling action of the wrist" (as described by Humle et al., 2011 for wild chimpanzees). 
339 Fig 2.

340 It may still be of some interest that, despite the focus of our study having been the scooping action, other

341 steps of the wild algae scooping sequence were also recorded in our study. The basic sequences of the

342 wild and our captive chimpanzees are very similar, although divergence exists between the order of some

343 steps, with Bossou chimpanzees first modifying their sticks before inserting them into the water (most

344 likely because they were detached directly from the tree or bush). Whilst the chimpanzees in our study

345 were also observed to modify their sticks, they did so less frequently than their wild counterparts. Since

346 the subjects in this study were provided with already detached sticks, they did not need to modify the

347 length of the sticks; at least not as often as wild chimpanzees (and, as the sticks provided were already

348 around the same length as that recorded in Bossou (Humle et al., 2011), further modification was not

349 often necessary). A total of four instances of stick modification were recorded throughout our six testing

350 sessions - that is, stick modification happened in $30 \%$ of all retrieval attempts (including unsuccessful

351 ones). All modifications occurred after the sticks were first inserted into the water. In all these instances

$352 \mathrm{HO}$ and LO used their fingers or teeth to break off a small piece of the stick, perhaps to make it into a

353 more manageable length to retrieve the bread crusts that had floated too close to the mesh (all instances of

354 stick modification occurred when the crusts were closest to the mesh, see S1 Fig for stills on the stick

355 modification method).

356 Additional techniques

357 Due to slight differences in the overall physical setup between our experiment and the wild, we expected

358 that the chimpanzees in our study would show additional new behaviours. This was indeed the case, and

359 both $\mathrm{HO}$ and LO were observed to occasionally make use of the sides of the water container to retrieve

360 the bread crusts. The basic sequence of this 'side technique' was as follows: first, the stick was placed on

361 the upper part of the bread crust, which was then pushed towards one of the sides of the bucket. Then,

362 pressure was placed on the crust to slide it up the edge of the container and onto the rim. Once the bread 
363 was on the rim, it was pulled towards the mesh and retrieved with the fingers (see S2 Fig for camera stills

364 of this method). All side technique attempts to retrieve the bread pieces were also coded. In both subjects,

365 the scooping technique was more commonly used than the side technique: in HO 68.9\% (20/29) of

366 attempts were with the scooping technique and 31.1\% (9/29) of the attempts were with the side technique.

367 In LO $61.8 \%(55 / 89)$ of the attempts to retrieve the bread crust were carried out using the scooping

368 technique and $38.2 \%(34 / 89)$ were using the side technique.

\section{Success Levels}

371 In all three trials, both $\mathrm{HO}$ and LO retrieved all three pieces of bread crust (including small pieces which

372 resulted from some disintegration of the bread crusts) within a maximum of six minutes. Mean retrieval

373 time for each bread piece using the scooping technique in Group 1 (HO) was 4sec ( $\mathrm{SD}=1 \mathrm{sec})$; in Group 2

374 (LO): $8 \mathrm{sec}(\mathrm{SD}=3 \mathrm{sec}$; recorded from when the tool came in contact with the piece to when the individual

375 started to feed). Mean retrieval time using the side technique in Group $1(\mathrm{HO})$ was $20 \mathrm{sec}(\mathrm{SD}=12 \mathrm{sec})$; in

376 Group 2 (LO): $7 \mathrm{sec}(\mathrm{SD}=2 \mathrm{sec})$.

\section{Individual Variation in Scooping Technique}

378 Individual variations in scooping technique were observed in the wild (Humle et al., 2011). Most

379 frequently, Bossou individuals held the tool between the thumb and the index finger when scooping, but 380 occasionally some gripped the tool between their middle and index fingers - although the exact number of 381 times each variant occurred was not reported (Humle et al., 2011). Additionally, after scooping, some 382 chimpanzees fed on the algae directly from the stick, whilst others, more rarely, gathered the algae off the 383 stick with their fingers and then licked it off their hands. As in the wild, there were also individual 384 differences between grips and feeding methods in our test subjects. To identify potential individual 385 differences we coded all clear cases of finger positioning and feeding methodologies for HO and LO 
386 (instances were not coded if the video was not clear enough to identify grip or feeding method). Table 1

387 shows the frequencies of these variants between $\mathrm{HO}$ and LO.

388 Table 1.

389 As can be seen in Table 1, HO varied continuously between grips, and showed no preference for the 390 middle and index grip whilst LO showed some preference for holding the stick between the thumb and 391 index finger, similarly to Bossou chimpanzees. Furthermore, HO occasionally used the stick directly to 392 feed, but preferred to use her fingers. LO only used her fingers to feed. Thus, overall, a comparable range 393 of individual differences to wild chimpanzees were observed in this study.

\section{Discussion}

395 Our results demonstrate that the wild form of scooping behaviour re-appeared independently in two naïve 396 chimpanzees (it was reinnovated twice). Thus, unlike human cumulative cultural behaviour, the observed patterns of scooping behaviour in the wild can be explained via Socially Mediated Serial Reinnovations (SMSR), rather than requiring high fidelity social learning mechanisms. As the scooping behaviour was independently reinnovated by two naïve chimpanzees, this fulfils the most conservative requirement for a latent solution (the double-case ZLS standard), and it strongly suggests that chimpanzees elsewhere also have the potential to produce this behaviour individually (though they may of course still be socially

402 influenced in, e.g. where to feed and what to feed on when using this technique). Scooping behaviour is a 403 latent solution in chimpanzees.

404 Given these findings, a latent solution account is not only probable for the first chimpanzee(s) who 405 innovates the scooping behaviour in a particular group (e.g. by beginning to eat surface algae using a 406 tool), but also for those who then "join in" due to low-fidelity social learning. The type of social learning 407 used is most likely one that utilises each chimpanzee's ability to reinnovate the behaviour - but does not 408 transmit the behavioural form itself (i.e. the social learning is not of high-fidelity type). Thus, our results 
409 strongly suggest that each individual chimpanzee is capable of reinnovating the behaviour independently,

410 and that for those surrounded by others who already have expressed the behaviour, low-fidelity social

411 learning mechanisms simply facilitate their own expression of this behaviour - increasing (and

412 harmonizing) the frequency of individuals reinnovating the behaviour in the population (SMSR).

413 As a thought experiment, if we were to imagine all forms of social learning - including low-fidelity social

414 learning - were completely absent from all chimpanzees, following the ZLS logic, behaviours such as

415 scooping would still re-appear (though in many cases, rarely), given the right circumstances ${ }^{5}$. Indeed,

416 scooping in the wild has also been reported outside the potential "cultural reach" of Bossou (Humle et al.,

417 2011), namely in Odzala National Park, Congo (around 3,000km apart; Devos et al., 2001). Why then, do

418 we not see more populations engaged in algae (or other food) scooping? Perhaps this is due to local trade-

419 offs between the necessity and the opportunity hypothesises (e.g. Fox et al., 1999), a possible explanation

420 for the fact that most wild innovations never "catch on" (Nishida et al., 2009) i.e. never lead to SMSRs

421 (more on this below).

422 This study provided evidence that chimpanzee scooping, a tool-use behaviour, is a latent solution (just

423 like other (non-tool-use) great ape behaviours that have been tested following the Latent Solution Test

424 methodology (Tennie et al., 2009; Tennie et al., 2008; Allritz et al., 2013; Menzel et al., 2013; Reindl et

425 al., 2016). In its current, strong formulation, the ZLS hypothesis makes a clear prediction: every wild-type

426 non-human great ape behaviour should reappear in at least some subjects of the same species ${ }^{6}$ who are

427 naïve to the behaviour in question when tested in Latent Solution Test settings (Tennie et al. 2009;

428 Henrich \& Tennie, in press). If this is the case, then human and chimpanzee cultures are ultimately

\footnotetext{
5 The likelihood of innovation is influenced by ecological (e.g. presence of algae) and behavioural ecological conditions (e.g. the nutritional need for algae in a given population - and these needs may differ between populations based on their ecology and their already adopted latent solutions). For more detail on the opportunity and necessity hypothesises see, e.g., Fox et al. (1999)).

${ }^{6}$ Though note that sometimes these behaviours also appear in different species, due to phylogeneticallyshared parts of their ZLS.
} 
429 founded on different underlying mechanisms ${ }^{7}$. Over time, this dissimilarity leads to very different 430 downstream effects: a restriction to behaviours drawn from the individually-bounded "Zone of Latent

431 Solutions" in chimpanzees versus the open-endedness of cumulative culture in humans (Tennie et al., 432 2009; although note that despite possessing extensive social learning abilities, human children are 433 surprisingly poor innovators, e.g. Beck et al., 2011; Nielsen 2013 but see also Reindl et al., 2016; Nelder 434 et al., 2017).

435 Note that we are not claiming that chimpanzee tool-use behavioural forms are genetic, in the sense that 436 they have been directly selected for by natural selection. We do not envision a genetic structure that 437 directly encodes scooping behaviour. Instead, apes have specialised in enhanced individual learning, i.e. 438 in innovations - and, at least for chimpanzees (Whiten et al., 1999; Whiten et al., 2001) and orangutans 439 (van Schaik et al., 2003), this is already well expressed by their varied use of tools in the wild (the other 440 great apes showcase their skills, too, but do this more so in captivity). The unspecialised, low-fidelity 441 social learning mechanisms that apes use are piggybacking on these innovative powers (we hypothesize 442 that ape cultures are based (perhaps in their entirety) on such socially mediated individual reinnovations).

443 In this synergy between individual and social learning, apes do not seem to be very special - indeed, 444 social and individual learning is highly correlated across the primate range (Reader \& Laland, 2001). Yet, 445 in their absolute levels of complexity they can reach in this way (e.g. see the case of nutcracking - but 446 also the sheer number of different tool uses that are thus enabled), great apes are exceptional animals 447 (alongside some bird species; e.g. Weir \& Kacelnik, 2006; Rutz et al., 2016).

\section{Target scooping action}

449 This study focused on the scooping action, the target behavioural form for which we examined the role of 450 social versus individual learning in its emergence. Both wild (Humle et al., 2011) and naïve chimpanzees 451 (this study) show this behavioural form (in particular, they rotate their wrist to wrap the food around the

\footnotetext{
${ }^{7}$ Humans have their own ZLS; Vygostky, 1978; Reindl et al, 2016; Reindl et al. in press, but can and do
} copy the forms of behaviours outside their ZLS, Tomasello, 1999; Henrich, 2015. 
452 tool, before retracting it towards them). Our study suggests that this technique is rather easily reinnovated

453 by individual chimpanzees, given a) the speed with which they expressed the technique, b) that two

454 subjects did so and c) that none of our successful test subjects had an opportunity to observe this 455 behaviour previously or during testing. Thus, our data renders it parsimonious to assume that the 456 scooping technique in the wild also arises on an individual level - as a latent solution.

457 Our conclusion is notwithstanding the fact that great apes in captivity have been shown to be generally 458 more proficient and/or motivated to use tools than those living in the wild, a phenomenon known as 'the 459 captivity effect' (Tomasello \& Call, 1997; van Schaik et al., 1998). The captivity effect does not impact 460 our findings - or indeed any other latent solution experiment outcome - as the effect merely increases the 461 likelihood of individual expression, but the effect does not prescribe the behavioural form itself. To the 462 best of our knowledge, the ZLS approach is best suited in providing an explanation for the similarities in 463 behavioural forms that are observed across independent individuals - as for example in the present study.

\section{Individual differences}

465 Individual differences in single actions during scooping behaviour observed in Bossou chimpanzees have 466 seemingly been suggested as evidence for social learning: "Individual variations in the different algae467 feeding techniques described here also should be further explored. The patterns of intracommunity 468 patterns of algae-feeding techniques may correlate with observational learning [...] and thus purport a 469 social learning mechanism in their transmission" (Humle et al., 2011). However, comparable differences 470 in action-level techniques were also found between our captive subjects - despite the fact that our subjects 471 could not have observed the Bossou chimpanzees. The existence of these small individual differences 472 shown by naïve chimpanzees in this study suggests that these differences are also a product of individual, 473 rather than social, learning. In general, a more convincing argument for social learning in the wild would 474 have been similarity of details of tool behaviour within a community but systematic differences between 475 groups (including to our study), unrelated to ecological and/or genetic differences. Currently the evidence 
476 for such variations in wild chimpanzees is limited (Langergraber et al., 2010), and even when such 477 differences are observed (Luncz \& Boesch, 2014), they do not reflect differences on the level of 478 behavioural form. The observed differences can instead be explained through low fidelity social learning 479 mechanisms such as stimulus enhancement (for example in explaining the use of wood hammers versus 480 stone hammers when nut cracking, as in Luncz \& Boesch, 2014).

481

482

484

485

486

487

488

489

490

491

492

493

494

495

496

497

498

499

\section{Potential objections}

Although only one chimpanzee in each group demonstrated the scooping behaviour targeted in this study (due to the apparatus being monopolised by these successful individuals) this is sufficient data to suggest that the behaviour is a latent solution for chimpanzees. Previously it was argued that it would suffice for only one individual to spontaneously show the behaviour for it to be considered within the species' ZLS and that even a single innovation would logically demonstrate that social learning is not necessary for it to occur (compare Tennie et al., 2009). In our study, we observed the spontaneous reinnovation of scooping actions not only in one, but in two, independent chimpanzees, who never received any relevant demonstrations, training or experiences, thus fulfilling the even more stringent requirements for the double-case ZLS standard, which we propose for relatively less complex behaviours as the one tested here (see above).

In no way does our data negate a role of low-fidelity social learning in scooping, or any other chimpanzee behaviour when looking at the population level. Indeed, (low-fidelity) social learning mechanisms likely homogenise the likelihood of individual learning of many chimpanzee behaviours, and therefore (though not explaining the actual form of the behaviours in question) can play a decisive role in explaining the distribution of these behaviours as they are observed in ape populations. Once an individual has innovated the behaviour, several low fidelity forms of social learning can help homogenise this behaviour across the population, by way of sustaining a chain reaction, where each single reaction consists of an individual reinnovation (leading to the final "cultural pattern" observed in the wild; Whiten et al., 1999; Whiten et 
500 al., 2001). The only caveat to this domino-effect explanation is that not every latent solution will actually

501 "catch on" once it is innovated in a given group (most innovations in wild chimpanzees do not, in fact, 502 spread; Nishida et al., 2009). The main reason for innovations not spreading in the wild are not altogether 503 clear yet, but may be related to the fact that wild apes are rather neophobic and thus de facto unlikely to 504 adopt behavioural forms (contra the captivity effect for captive apes; Forss et al., 2015), and so are 505 unlikely to reinnovate. In addition, perhaps meta-rules (so-called social learning strategies) exist in apes 506 for when to apply their low-fidelity social learning mechanisms, which might act against the usually 507 observed type of wild innovators being influential (Kendal et al., 2015). And if, in addition, current 508 claims for a majority influence in chimpanzees (Luncz \& Boesch, 2014) can be further substantiated, then 509 these effects could also proof detrimental to the uptake of latent solutions. Of course, latent solutions that 510 are new to a wild group of apes sometimes do become population-wide behaviours after they are 511 innovated (or at least occur lastingly across several individuals, see Lamon et al., 2017) - but this happens 512 rarely and (currently, at least) unpredictably. Nevertheless, this alternative ZLS view explains the 513 population differences we see across ape populations at least as well as the currently more widespread 514 high-fidelity social learning account. In addition, the ZLS explanation stands alone in not requiring the 515 additional assumption that apes are able to socially learn with high fidelity (an ability that they may well 516 lack, see introduction).

517 Some objections on the results of this study may still remain. Firstly, some may claim that one cannot 518 fully discount that the chimpanzees in this study saw demonstrations of the scooping action previous to 519 our test. Given that apes are long-living animals, and not observed 24 hours a day, the field has little hope 520 to ever be able to negate such ad hoc claims (see also introduction). However, we must ask how likely it 521 actually is that the chimpanzees were exposed to a similar behaviour before testing, given that the keepers 522 all (and independently) confirmed that subjects were naïve to scooping. This is especially the case here, 523 where we detected scooping in two groups - thus, the behaviour would have had to remain unobserved by 524 the keepers in not just one, but both groups of chimpanzees. 
525 The chimpanzees in our study did have access to sticks before testing, which some might argue threatens

526 their naivety to parts of the task. However, in this study we merely follow the accepted standard in field

527 studies, in which, despite similarities in tools and actions, each chimpanzee behaviour is classified

528 separately (see, for example: 'ant fish' and 'termite fish', both of which involve the same tool and action,

529 but the different food sources being accessed is used to qualify them as separate behaviours; Whiten et al.,

530 2001). The aim of our study was not to assess the tool-use abilities of completely stick-naïve

531 chimpanzees, (also because it is practically impossible to find stick-tool-naïve chimpanzees in captivity),

532 but rather to assess whether chimpanzees who are naïve to the scooping behavioural form, as described in

533 the literature (Whiten et al., 1999; 2001; Humle et al., 2011) would demonstrate the same behavioural

534 form in the absence of social learning opportunities. Thus, whilst the chimpanzees in this study had

535 experience with sticks, they were never faced with the problem of having to retrieve floating out-of-reach

536 food inside a body of water. Importantly, the chimpanzees were naïve to the 'swivelling' wrist action

537 required for the behaviour, i.e. to the key part of our target behavioural form (Humle et al., 2011).

538 Therefore, the subjects were naïve to the main aspect of the task - i.e. the target behaviour - making them

539 ideal candidates to assess whether they would spontaneously solve the problem in a similar way to their

540 wild counterparts.

541 Furthermore, it might also be objected, "extraordinary claims require extraordinary data". The

542 extraordinary claim in our case might then be argued to be that scooping represents a latent solution, not

543 necessitating social learning to emerge across individuals. We agree with the notion that extraordinary

544 claims do require extraordinary evidence. However, 'extraordinary' evidence supporting the latent

545 solution hypothesis in apes has already been provided (see also list above): naïve chimpanzees and

546 bonobos independently reinnovated leaf swallowing behaviour (Huffman \& Hirata, 2004; Menzel et al.,

5472013 ) despite never observing the behaviour in others before testing (and one can be $100 \%$ sure of this, as

548 the leaf swallowing behavioural form occurs within the mouth area, and is therefore entirely concealed to

549 observers). Therefore, our claim that a given great ape behaviour represents a latent solution must no 
550 longer be regarded an extraordinary claim. Following the same logic as these previous studies, our study -

551 demonstrating two independent cases of reinnovation across two separate groups - therefore provides

552 conclusive evidence supporting the notion that scooping lies within chimpanzees' ZLS.

\section{Conclusion}

554 This research extends and supports previous work on the ZLS in great apes (Tennie et al., 2009; Tennie et

555 al., 2008; Allritz et al., 2013; Menzel et al., 2013; Reindl et al., 2016) which also found that other wild type ape behaviours develop spontaneously in naïve individuals and do not depend on social transmission, yet ours is the first study (to the best of our knowledge), to apply the latent solution logic explicitly to a chimpanzee tool-use behaviour. Examining tool-use behaviours is especially relevant for the study of non-human great ape cognition and evolution and also for understanding the evolution of human material rely on social transmission can aid in the reconstruction of the evolution of hominin tools, which we believe may also have been characterised (at least for a long time) by individual reinnovations sensu the ZLS hypothesis (Tennie et al., 2016; Tennie et al., in press).

Our study also highlights the importance of re-evaluating chimpanzee cultures in the light of latent solutions (Tennie et al., 2009). The classic method of exclusion (Whiten et al., 1999; Whiten et al., 2001; van Schaik et al., 2003; Robbins et al., 2016), which detects behaviour patterns across wild populations, has many commendable points and has sparked a flurry of research into animal culture. However, it is likely that many or all of these behaviour patterns come about via a combination of several factors, such as genetics, ecological and cultural factors (Laland \& Janik, 2006; and in our view, the latter consisting of low fidelity social learning that is ultimately fuelled by individual learning). It is important to delve

571 further into the underlying mechanisms of each behaviour by submitting them to latent solution testing as

572 in the current study, especially before assigning them cultural status in the modern human sense of the 573 word. Whiten (2000) best embodied this approach when stating: "the nature of the cognitive process of 
574 transmission matters in understanding what kinds of traditions, or cultures, really operate among 575 nonhuman primates". We could not agree more.

576 Our latent solution approach for tool-use is new to great apes - at least when applied to wild type 577 behaviours - but has already been tested in other species (for example, with New Caledonian crows 578 (Kenward et al., 2005) and woodpecker finches (Tebbich et al., 2001)). Using the latent solution 579 methodology - providing naïve individuals who have never had the opportunity to see or learn from 580 others with the ecological set-up of materials and reward structures - will further aid in identifying the

581 necessary underlying mechanisms and their relative roles in the expression of that behaviour. Following 582 this process we can better understand what forms of culture exist in both human and non-human animals 583 - and which factors are shared and which are not. In this study we found that scooping is within 584 chimpanzees' Zone of Latent Solutions and therefore is not indicative of high fidelity social learning. 585 With more research in the field following the latent solutions method, we predict that several other 586 behaviours, including those that were previously believed to require social learning (e.g. Whiten et al., 587 1999; Whiten et al., 2001) may soon follow suit. 


\section{Acknowledgments}

594 The authors are very grateful to Twycross Zoo for their kind cooperation and help for this research, in 595 particular: Charlotte Macdonald, Simon Childs, Zak Showell, Sharon Redrobe, as well as all the animal 596 keepers involved. The authors also thank Flo Rocque for her help collecting the data, Alice Coombes and 597 Clare Williams for coding, and Eva Reindl and Zanna Clay for their comments on earlier drafts of the 598 manuscript. We are grateful to the anonymous reviewers for their thorough and helpful comments on 599 earlier drafts of the manuscript.

600

601

602 
604 Beck SR, Apperly IA, Chappell J, Guthrie C, Cutting N. (2011). Making tools isn't child's play.

605 Cognition, 119(2), 301-306. https://doi.org/10.1016/j.cognition.2011.01.003

606

607 Boesch C, Marchesi P, Marchesi N, Fruth B, Joulian F. (1994). Is nut cracking in wild chimpanzees a

608 cultural behavior? Journal of Human Evolution, 26(4), 325-338. https://doi.org/10.1006/jhev.1994.1020

609

610 Boesch C, Kalan AK, Agbor A, Arandjelovic M, Dieguez P, Lapeyre V, Kuhl HS. (2016). Chimpanzees

611 routinely fish for algae with tools during the dry season in Bakoun, Guinea. American Journal of

612 Primatology pre-print. Doi: 10.1002/ajp.22613.

613

614 Boesch C. (1996). The emergence of cultures among wild chimpanzees. In: Runciman WG, Smith JM, 615 Dunbar RIM (Eds.), Evolution of Social Behaviour Patterns in Primates and Man (pp. 251-268). New 616 York, USA: Oxford University Press.

617

618 Boyd R, \& Richerson PJ. (2005). The origin and Evolution of Cultures. Oxford University Press. Oxford: 619 Oxford University Press. https://doi.org/10.1016/S0169-5347(97)84100-6

620

621 Byrne RW, Corp N, Byrne JME. (2001). Estimating the complexity of animal behaviour: How mountain 622 gorillas eat thistles. Behaviour, 138(4), 525-557. Doi: 10.1163/156853901750382142

623

624 Caldwell CA, \& Millen AE. (2009). Social learning mechanisms and cumulative cultural evolution: Is 625 imitation necessary? Psychological Science, 20(12), 1478-1483. https://doi.org/10.1

626

627 Collias E, Collias N. (1964). The development of nest-building behaviour in a weaverbird. The Auk. 81, 628 42-52. Doi: 10.2307/4082609. 
629

630 Custance D, Whiten A, Fredman T. (1999). Social learning of an artificial fruit task in capuchin monkeys 631 (Cebus apella). Journal of Comparative Psychology, 113(1), 13-23. https://doi

632

633 Custance D, Whiten A, Sambrook T, Galdikas B. (2001). Testing for social learning in the artificial fruit 634 processing of wildborn orangutans (Pongo pygmaeus), Tanjung Puting, Indonesia. Animal Cognition, 635 4(3-4), 305-313. https://doi.org/10.1007/s100710100100

636

637

De Waal F. (2001) The ape and the sushi master, reflections of a primatologist. London, UK: Basic 638 Books.

639

640

Dean LG, Kendal RL, Schapiro SJ, Thierry L. (2011). Identification of the Social and Cognitive 641 Processes Underlying Human Cumulative Culture. Science, 3801(1974), 23-26.

642

643 Devos C, Gatti S, Levrero F. (2002). New record of algae feeding and scooping by pan t. troglodytes at 644 Lokoue Bai in Odzala National Park, Republic of Congo. Pan African News, 9, 19-21.

645

646

Dindo M, Thierry B, Whiten A. (2008). Social diffusion of novel foraging methods in brown capuchin 647 monkeys (Cebus apella). Proceedings. Biological Sciences / The Royal Society, 275

648

649 Forss SIF, Schuppli C, Haiden D, Zweifel N, van Schaik CP. (2015). Contrasting responses to novelty by 650 wild and captive orangutans. American Journal of Primatology, 77(10), 1109-1121. Doi:

651 10.1002/ajp.22445.

652

653 Fox EA, Sitompul AF, van Schaik CP. (1999). Intelligent tool use in Sumatran orangutans. In: Parker ST, 654 Mitchell RW, Miles HL (Eds.), The mentalities of gorillas and orangutans (pp. 99 - 116). Cambridge, 655 UK: Cambridge University Press. 
656

657 Fragaszy DM, Izar P, Visalberghi E, Ottoni EB, DeOliveira MG. (2004). Wild capuchin monkeys (Cebus 658 libididinosus) use anvils and stone pounding tools. American Journal of Primatology, 64, 359-366. Doi: 659 10.1002/ajp.20085.

660 Gruber T, Clay Z, Zuberbuhler K. (2010). A comparison of bonobo and chimpanzee tool use: Evidence 661 for a female bias in the Pan lineage. Animal Behaviour, 80(6), 1023-1033. Doi:

662 http://dx.doi.org/10.1016/j.anbehav.2010.09.005.

663

664 Henrich J., \& Tennie C. (in press). Cultural Evolution in Chimpanzees and Humans. Working Paper, 166531.

666

667 Henrich J. (2015). The secret of our success: how culture is driving human evolution, domesticating our 668 species, and making us smarter. Oxford, UK: Princeton University Press.

669

670

Herrmann E, Call J, Hernàndez-Lloreda MV, Hare B, Tomasello M. (2007). Humans have evolved

671 specialized skills of social cognition: the cultural intelligence hypothesis. Science, 317(5843), 1360-1366.

672

673 Hobaiter C, Poisot T, Zuberbuhler K, Hoppitt W, Gruber T. (2014). Social network analysis shows direct 674 evidence for social transmission of tool use in wild chimpanzees. Plos Biology 12(9). Doi:

675 10.1371/journal.pbio.1001960.

676

677 Hopper LM, Spiteri A, Lambeth SP, Schapiro SJ, Horner V, Whiten A. (2007). Experimental studies of 678 traditions and underlying transmission processes in chimpanzees. Animal Behaviour, 73(6), 1021-1032.

679

680 Hopper LM. (2016). The individual and social drivers of primate innovation. PeerJ Preprints 4:e2276v1. 681 Doi: https://doi.org/10.7287/peerj.preprints.2276v1. 
682

683 Huffman MA, Hirata S. (2004). An experimental study of leaf swallowing in captive chimpanzees:

684 insights into the origin of a self-medicated behavior and the role of social learning. Primates, 45(2), 113 -

685 118. Doi: 10.1007/s10329-003-0065-5.

686

687 Humle T, Yamakoshi G, Matsuzawa T. (2011). Algae scooping remains a puzzle. In: Matsuzawa T,

688 Humle T, Sugiyama Y. (Eds.), The chimpanzees of Bossou and Nimba (pp. 117-122). Tokyo, JP:

689 Springer Verlag.

690

691 Kendal R, Hopper LM, Whiten A, Brosnan SF, Lambeth SP, Schapiro SJ, Hoppitt W. (2015).

692 Chimpanzees copy dominant and knowledgeable individuals: implications for cultural diversity Evolution 693 of Human Behaviour, 36(1), 65-72. Doi: http://dx.doi.org/10.1016/j.evolhumbehav.2014.09.002

694

695 Kenward B, Weir AS, Rutz C, Kacelnik A. (2005). Behavioural ecology: tool manufacture by naïve 696 juvenile crows. Nature, 433, 121. Doi: 10.1038/433121a.

697

698 Kis A, Huber L, Wilkinson A. (2016). Social learning by imitation in a reptile (Pogona vitticeps). Animal 699 Cognition, 18(1), 325-331. Doi: 10.1007/s10071-014-0803-7.

700

701 Koops K, Furuichi T, Hashimoto C. (2015). Chimpanzees and bonobos differ in intrinsic motivation for 702 tool-use. Nature Scientific Reports, 5. Doi: 10.1038/srep11356.

703

704 Koops K, Visalberghi E, van Schaik PC. (2014). The ecology of primate material culture. Biology 705 Letters, 10, 1-4. Doi: 10.1098/rsbl.2014.0508. 
707 Kline MA. (2014). How to learn about teaching: An evolutionary framework for the study of teaching

708 behavior in humans and other animals. Behavioral and Brain Sciences, 38.

709

710 Kummer H, Goodall J. (1985). Conditions of Innovative Behaviour in Primates. Philosophical

711 Transactions of the Royal Society of London. Series B.

712

713 Laland NK, Janik MV. (2006). The animal cultures debate. Trends in Ecological Evolution, 21, 542-547.

714 Doi: 10.1016/j.tree.2006.06.005.

715

716 Lamon N, Neumann C, Gruber T, Zuberbühler K. (2017). Kin-based cultural transmission of tool use in

717 wild chimpanzees. Science Advances, 3(4), 1-10. https://doi.org/DOI: 10.1126/sci

718

719 Langergraber KE, Boesch C, Inoue E, Inoue-Murayama M, Mitani JC, Nishida T, Pusey A, Reynolds V, 720 Schubert G, Wrangham RW. (2010). Genetic and 'cultural' similarity in wild chimpanzees. Proceedings 721 of the Royal Society B. Doi: 10.1098/rspb.2010.1112.

722

723 Lefebvre L. (1986). Cultural Diffusion of a Novel Food-finding Behaviour in Urban Pigeons: an 724 Experimental Field Test. Ethology, 71(4), 295-304. https://doi.org/10.1111/j.1439-0310.1986.tb0059

725

726

727

728

729

730 Luncz LV, Boesch C. (2014). Tradition over trend: neighbouring chimpanzee communities maintain

731 differences in cultural behaviour despite frequent immigration of adult females. American Journal of

732 Primatology, 76(7), 649-657. Doi: 10.1002/ajp.22259. 
734 McGrew WC. (1998). Culture in nonhuman primates? Annual Review of Anthropology, 27, 301-328.

735

736 Menzel C, Fowler A, Tennie C, Call J. (2013) Leaf surface roughness elicits leaf swallowing in captive 737 chimpanzees (Pan troglodytes) and bonobos (P.paniscus) but not in gorillas (Gorilla gorilla) or orang 738 utans (Pongo abelii). International Journal of Primatology, 34(3), 533-553. Doi: 10.1007/s10764-013$7399679-7$.

740

741 742

743

744 Musgrave S, Morgan D, Lonsdorf E, Mundry R, Sanz C. (2016). Tool transfers are a form of teaching 745 among chimpanzees. Scientific Reports, 6. Doi:10.1038/srep34783.

746

747 Myowa-Yamakoshi M, \& Matsuzawa T. (2000). Imitation of intentional manipulatory actions in 748 chimpanzees (Pan troglodytes). Journal of Comparative Psychology, 114(2), 381-391.

749

750 Neldner K, Mushin I, \& Nielsen M. (2017). Young children's tool innovation across culture: Affordance 751 visibility matters. Cognition, 168, 335-343. https://doi.org/10.1016/j.cognition.2017.07.015

752

753 Nielsen M. (2013). Young children's imitative and innovative behaviour on the floating object task.

754 Infant and Child Development, 22(1), 44-52. https://doi.org/10.1002/icd.1765

Moore R. (2012). Social learning and teaching in chimpanzees. Biological Philosophy, 28, 879-901.Doi: 10.1007/s10539-013-9394-y. 
756 Nishida T, Matsusaka T, McGrew WC. (2009). Emergence, propagation or disappearance of novel

757 behavioural patterns in the habituated chimpanzees of Mahale: A review. Primates, 50(1), 23-36. Doi:

758 10.1007/s10329-008-0109-y.

759

760 Perry ES. (2006). What cultural primatology can tell anthropologists about the evolution of culture.

761 Annual Review of Anthropology, 35, 171-190. Doi: 10.1146/annurev.anthro.35.081705.123312.

762

763 Reader SM, Laland KN. (2001). Social intelligence, innovation, and enhanced brain size in primates.

764 PNAS, 99(7), 4436-4441. Doi:10.1073/pnas.062041299.

765

766 Reader SM, \& Laland KN. (2003). Animal Innovation: An Introduction. In Animal Innovation (pp.3-

767 36). Oxford University Press. https://doi.org/10.1093/acprof:oso/9780198526223.003.0001

768

769 Reindl E, Bandini E, Tennie C. (in press). The zone of latent solutions and its relation to the classics:

770 Vygotsky and Kohler. In: Di Paolo LD, d'Almeida AFA, Vincenzo FD (Eds.), Social cognition in non-

771 human primates and early Homo. Berlin, Germany: Springer.

772 Reindl E, Beck SR, Apperly IA, Tennie C. (2016). Young children spontaneously invent wild great apes' 773 tool-use behaviours. Proceedings of the Royal Society B, 283. Doi: 10.1098/rspb.2015.2402.

774

775 Reindl E, Apperly IA, Beck SR, Tennie C. (2017). Young children copy cumulative technological design

776 in the absence of action information. Scientific Reports, (April), 1-11. https://d

777

778 Rendell L, Whitehead H. (2001). Culture in whales and dolphins. Behavioural Brain Science, 24, 309-

779 324. Doi: 10.1017/S0140525X0100396X. 
781 Robbins MM, Ando C, Fawcett KA, Grueter CC, Hedwig D, Iwata Y, Lodwick JL, Masi S, Salmi R,

782 Stoinski TS. (2016). Behavioural variation in gorillas: evidence of potential cultural traits. Plos One, 783 11(9). Doi: 10.1371/journal.pone.0160483.

784

785 Rutz C, Klump BC, Komarczyk L, Leighton R, Kramer J, Wischnewski S, Sugasawa S, Morrissey MB, 786 James R, St Clair JJH. (2016). Discovery of a species-wide tool use in the Hawaiian crow. Nature, 787 537(7620), 403-407. Doi:10.1038/nature19103.

788

789

Sakamaki T. (1998). First record of algae-feeding by a female chimpanzee at Mahale. Pan African News, 790 $5,1-3$.

791

792

Sanz CM, Morgan DM. (2007). Chimpanzee tool technology in the goualougo triangle, Republic of 793 Congo. Journal of Human Evolution, 52, 420-433.

794

795

Stoinski TS, Wrate JL, Ure, N, Whiten A. (2001). Imitative learning by captive western lowland gorillas 796 (Gorilla gorilla gorilla) in a simulated food-processing task. Journal of Comparative Psychology 8(17).

797

798

Tebbich S, Taborsky M, Fessl B, Blomqvist D. (2001). Do woodpecker finches acquire tool-use by social 799 learning? Proceedings of Biological Science, 268, 2189-2193. Doi: 10.1098/rspb.2001.1738.

800

Tennie C, Premo LS, Braun DR, McPherron SP. (in press). Resetting the null hypothesis: early stone 801 tools and cultural transmission. Forum Article in: Current Anthropology.

802

803 Tennie C, Braun DR, Premo LS, McPherron SP. (2016). The island test for cumulative culture in 804 Paleolithic cultures. In: Haidle MN, Conard NJ, Bolus M, (Eds.), The Nature of culture: based on an 805 interdisciplinary symposium 'the nature of culture' (pp. 121-133). Netherlands: Springer.

806 
807 Tennie C, Call J, Tomasello M. (2006). Push or pull: imitation vs. emulation in great apes and human

808 children. Ethology, 112, 1159-1169. Doi: 10.1111/j.1439-0310.2006.01269.

809

810 Tennie C, Hedwig D, Call J, Tomasello M. (2008). An experimental study of nettle feeding in captive 811 gorillas. American Journal of Primatology. 70, 584-593. Doi: 10.1002/ajp.20532.

812

813 Tennie C, Call J, Tomasello M. (2009). Ratcheting up the ratchet: On the evolution of cumulative culture. 814 Philosophical Transactions of the Royal Society, 364, 2405-2415. Doi: 10.1098/rstb.2009.0052.

815

816 Tennie C. \& Over H. (2012). Cultural intelligence is key to explaining human tool use. Behavioral and 817 Brain Sciences. 35, 242-243

818

819 Tennie C, Call J, Tomasello M. (2012). Untrained Chimpanzees (pan troglodytes schweinfurthii) fail to 820 imitate novel actions. PLoS ONE, 7, e41548.

821

822 Tomasello M, Davis-Dasilva M, Camak L, Bard K. (1987). Observational learning of tool-use by young 823 chimpanzees. Human Evolution, 2(2), 175-183. https://doi.org/10.1007/BF02436405

824

825 Tomasello M, Kruger AC, Ratner HH. (1993). Cultural learning. Behavioral and Brain Sciences, 16(3), 826 495. https://doi.org/10.1017/S0140525X0003123X

827 Tomasello M, Call J. (1997). Primate Cognition. New York, USA: Oxford University Press.

828

829 Tomasello M. (1999). The cultural origins of human cognition. Harvard, MA: Harvard University Press. 830 
831 van Schaik CP, Deaner R, Merrill M. (1998). The conditions for tool use in primates: implications for the

832 evolution of material culture. Journal of Human Evolution, 36, 719-741. Doi: 10.1006/jhev.1999.0304.

833

834 van Schaik CP, Ancreanz M, Borgen G, Galdikas B, Knott CD, Singleton I, Suzuki A, Utami SS, Merrill

835 M. (2003). Orangutan cultures and the evolution of material culture. Science, 299, 102-105. Doi:

$83610.1126 /$ science.1078004.

837

838 van de Waal E, Renevey N, Favre CM, Bshary R. (2010). Selective attention to philopatric models causes

839 directed social learning in wild vervet monkeys. Proceedings of the Royal Society of London B:

840 Biological Sciences, 277(1691). Retrieved from

841 http://rspb.royalsocietypublishing.org/content/277/1691/2105.short

842

843 Visalberghi E. (1987). Acquisition of nut-cracking behaviour by 2 capuchin monkeys (Cebus apella).

844 Folia Primatologica, 49(3-4), 168-181. https://doi.org/10.1159/000156320

845

846 Vygotsky L. (1978) Mind in society: development of higher psychological processes. Harvard University 847 Press, Harvard: USA.

848

849 Weir AS, Kacelnik A. (2006). A new caledonian crow (Corvus moneduloides) creatively re-designs tools 850 by bending or unbending aluminium strips. Animal Cognition, 9, 317-334. Doi: 10.1007/s10071-006$8510052-5$.

852

853 Whiten A, Custance D, Gomez JC, Teixidor P, Bard KA. (1996). Imitative learning of artificial fruit 854 processing in children (homo sapiens) and chimpanzees (pan troglodytes). Journal of Comparative 855 Psychology, 110, 3-14.

856 
857 Whiten A. (1998). Imitation of the Sequential Structure of Actions by Chimpanzees (Pan troglodytes).

858 Journal of Comparative Psychology, 112(3), 270-281. https://doi.org/10.1037/0735-7036.112.3

859

860 Whiten A, Goodall J, McGrew W, Nishida T, Reynolds V, Sugiyama Y, Tutin C, Wrangham W, Boesch 861 C. (1999). Cultures in chimpanzees. Nature, 399, 682-685. Doi: 10.1038/21415.

862 Whiten A. (2000). Primate culture and social learning. Cognitive Science, 24, 477-508. Doi:

863 http://dx.doi.org/10.1016/S0364-0213(00)00027-6.

864

865 Whiten A, Goodall J, McGrew WC, Nishida T, Reynolds V, Sugiyama Y, Tutin CEG, Wrangham RW, 866 Boesch, C. (2001). Charting cultural variation in chimpanzees. Behaviour. 138, 1481-1516. Doi:

$86710.1163 / 156853901317367717$.

868

869 Whiten A, Horner V, Litchfield AC, Marshall-Pescini S. (2004). How do apes ape? Animal Learning and 870 Behaviour, 32(1), 36-52. Doi: 10.3758/BF03196005.

871

872 Whiten A, Horner V, de Waal FBM. (2005). Conformity to cultural norms of tool use in chimpanzees.

873 Nature, 437(7059), 737-740. https://doi.org/10.1038/nature04047

874

875 Whiten A, van Schaik CP. (2007). The evolution of animal 'cultures' and social intelligence.

876 Philosophical Transactions of the Royal Society, 362, 603-620. Doi: 10.1098/rstb.2006.1998.

877

878 Whiten A, \& Mesoudi A. (2008). Review. Establishing an experimental science of culture: animal social 879 diffusion experiments. Philosophical Transactions of the Royal Society of London. Series B, Bio 8 (34). 
881 Whiten A, McGuigan N, Marshall-Pescini S, Hopper ML. (2009). Emulation, imitation, over-imitation 882 and the scope of culture for child and chimpanzee. Philosophical Transactions of the Royal Society, 364, 883 2417-2428. Doi: 10.1098/rstb.2009.0069.

884

885 Whiten A, Schick K, Toth N. (2009). The evolution and cultural transmission of percussive technology: 886 integrating evidence from palaeoanthropology and primatology. Journal of Human Evolution, 57(4), 420887 435. Doi: http://dx.doi.org/10.1016/j.jhevol.2008.12.010.

888

889 Whiten A. (1998). Imitation of the Sequential Structure of Actions by Chimpanzees (Pan troglodytes). 890 Journal of Comparative Psychology, 112(3), 270-281. https://doi.org/10.1037/0735-7036.112.3

891

892 Yamamoto S, Humle T, Tanaka M. (2013). Basis for Cumulative Cultural Evolution in Chimpanzees:

893 Social Learning of a More Efficient Tool-Use Technique. PLoS ONE, 8(1), e55768.

894

895

896

897

898

899

900

901

902

903 


\section{Figure 1}

\section{Experimental set-up}

Container with bread crusts in the foreground and one of the sticks inside the enclosure (photograph by EB).

*Note: Auto Gamma Correction was used for the image. This only affects the reviewing manuscript. See original source image if needed for review. 


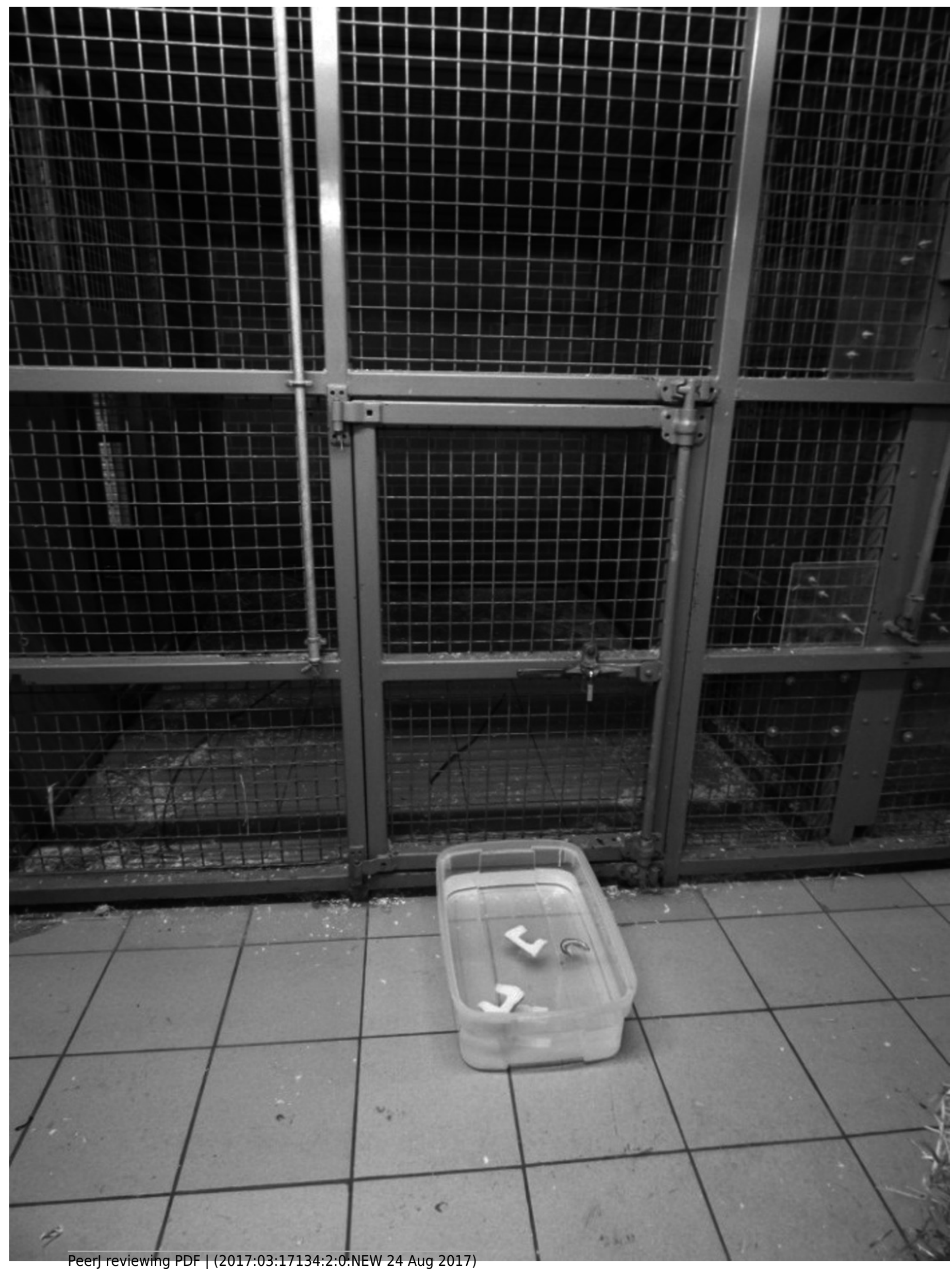




\section{Figure 2}

\section{Scooping sequence}

$\mathrm{HO}$ carrying out the scooping sequence. $\mathrm{A}$ : $\mathrm{HO}$ inserts the stick under the bread, $\mathrm{B}$ : using a 'swivelling' motion of the wrist, HO scoops up the bread (Humle et al., 2011) and C: HO retrieves the bread (camera stills by EB).

*Note: Auto Gamma Correction was used for the image. This only affects the reviewing manuscript. See original source image if needed for review. 

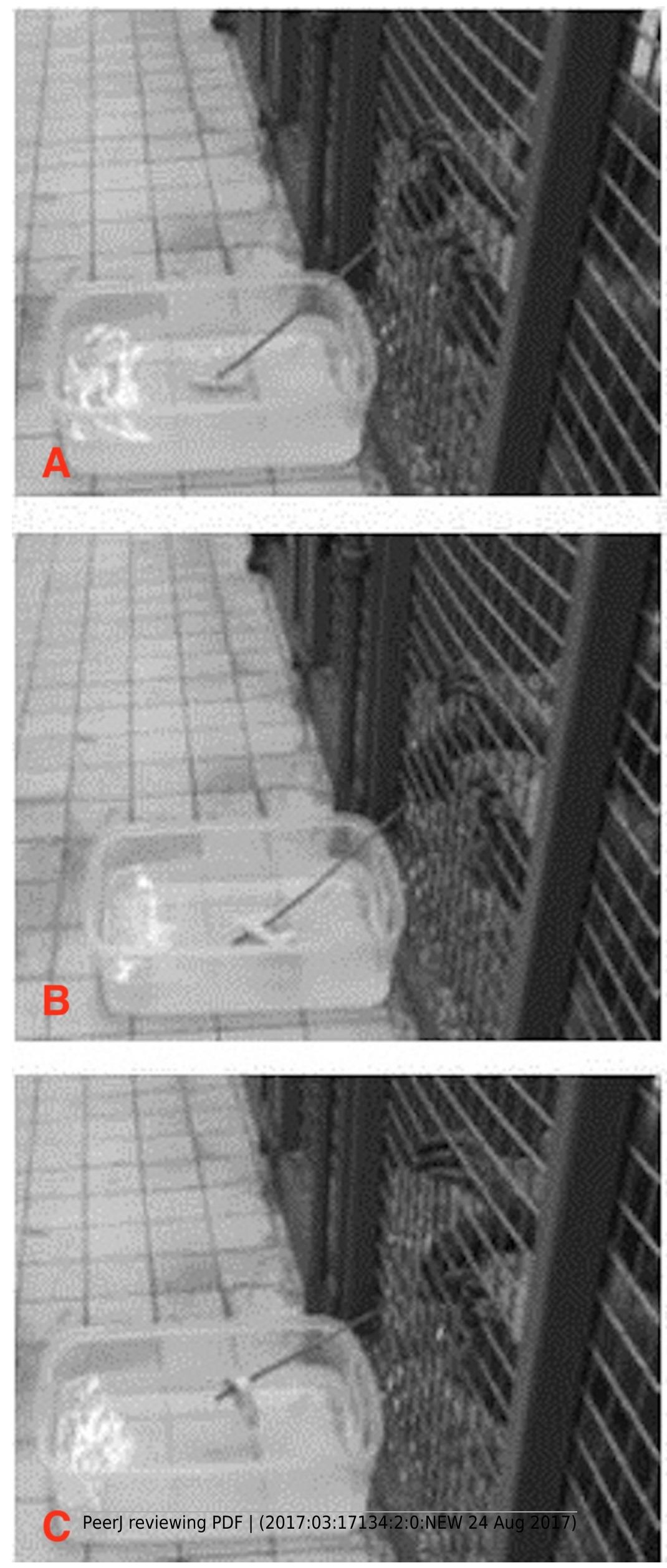


\section{Table $\mathbf{1}$ (on next page)}

Individual action variations

Number of times each action variant seen in the wild was performed by captive chimpanzees (only clearly visible instances were coded, including instances in which the stick was manipulated and no attempt was made). 
Wild Behaviour (Humle et al., 2011)

Stick held between thumb and index finger

Stick held between middle and index finger

Direct mouth feeding

Use of fingers to feed
HO/total

$22 / 45$

$23 / 45$

$8 / 21$

$13 / 21$
LO/total

$31 / 44$

$13 / 44$

$0 / 12$

$12 / 12$

1 Table 1. Number of times each action variant seen in the wild was performed by captive chimpanzees

2 (only clearly visible instances were coded, including instances in which the stick was manipulated and no 3 attempt was made).

4 